\title{
Line Formation in Be Star Disks: Shell Absorption and Rotation Law
}

\author{
W. Hummel \\ Institut für Astronomie und Astrophysik und Universitätssternwarte \\ München, Scheinerstr 1, D-81673 München, Germany \\ M. Vrancken \\ Royal Observatory of Belgium, Ringlaan 3, B-1180 Brussels, Belgium
}

Abstract. We constrain the rotation law in Be star circumstellar disks. We show that Keplerian rotation is a valid approximation.

\section{Introduction}

The rotation law in Be star circumstellar disks is usually parameterized by

$$
V_{\text {rot }}=V_{*} R^{-j},
$$

where $V_{*}$ is the inner boundary condition. $j=1$ holds for conservation of angular momentum and $j=\frac{1}{2}$ holds for Keplerian rotation. We will present two distinct methods to constrain $j$.

\section{Rotation Law from Line Profile Modeling}

$\mathrm{H} \alpha$ line formation in Be star disks is rather complex (e.g. Hummel 1997), however under certain conditions line formation becomes simpler. For Be stars with $\mathrm{H} \alpha$ emission lines of low equivalent width (EW) non-coherent scattering and electron scattering is no longer efficient, hence $\mathrm{H} \alpha$ line shapes are determined by the kinematical broadening. For this limit we apply the shear broadening model of Horne \& Marsh (1986) but extend their method by taking the finite size of the central star into account. This means we can study the impact of shell absorption and stellar obscuration on $\mathrm{H} \alpha$ line formation in a simplified manner.

Low EW $\mathrm{H} \alpha$ emission lines have been collected from several observing runs at OHP and Calar Alto and ESO (HR 5540 from Dachs et al. 1987) with $R=45000-72000$. Photospheric absorption profiles have been calculated and fit to the observations as by Vrancken et al. (1996). Net emission profiles (observed minus photospheric fit) are modeled varying the circumstellar density $n_{0}\left(R_{*}\right)$, the radial density power law index $m$, and outer disk radius $R_{\mathrm{d}}$. The inclination is estimated from $v \sin i$. Representative model fits are shown in Fig. 1. 

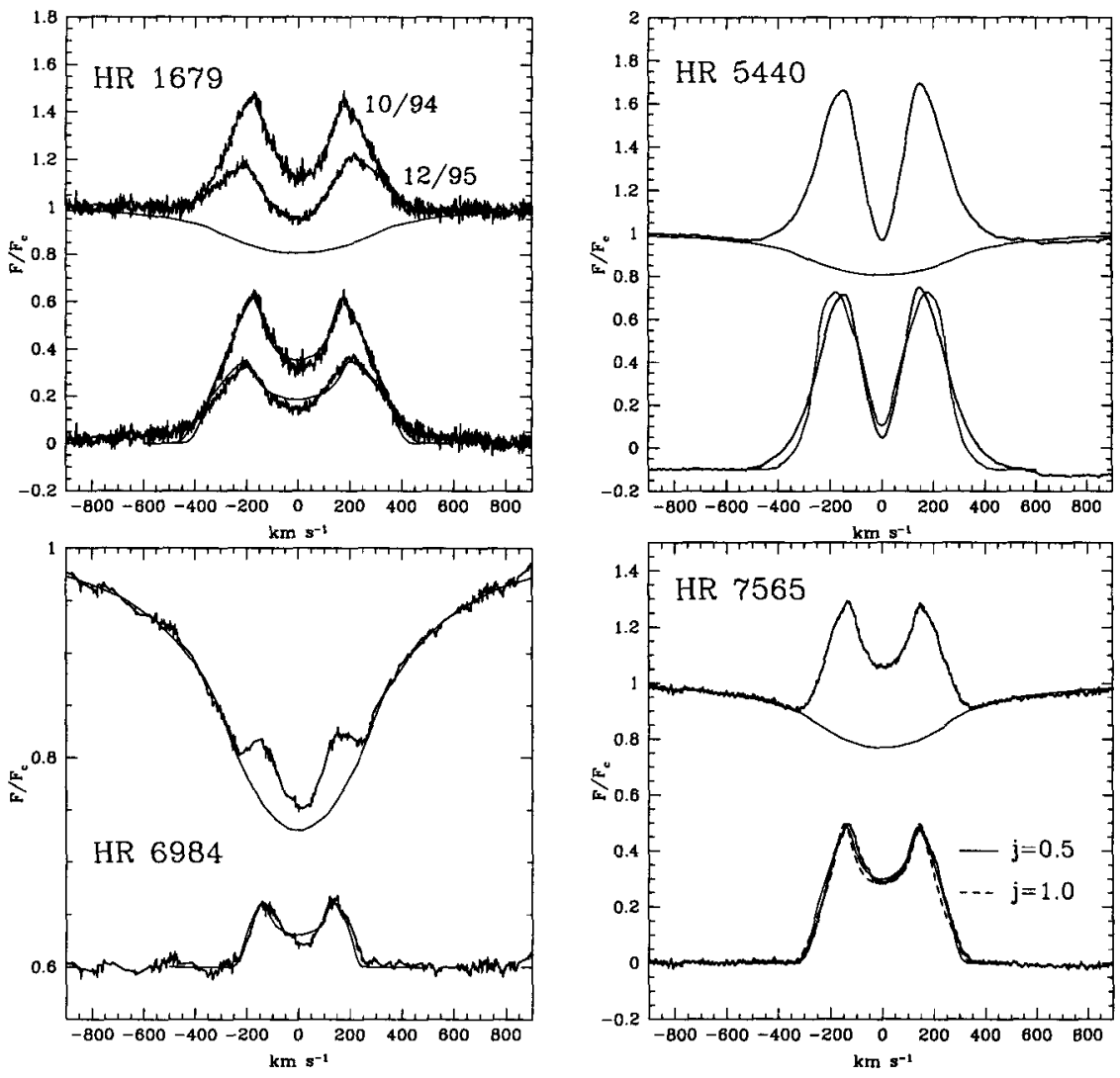

Figure 1. Low EW H $\alpha$ line profiles of four Be stars, theoretical stellar absorption profile (upper), net emission profile and model fit to the net emission (lower). If not otherwise noted, Keplerian rotation $j=\frac{1}{2}$ is used to fit the net emission.

Optically thin line profiles (e.g. HR 7565) can be fit by Keplerian rotation $\left(j=\frac{1}{2}\right)$ as well as by conservation of angular momentum $(j=1)$ and any value in between (Fig. 1). For Be stars seen at large inclinations (HR 5440) self absorption in the disk, shell absorption and stellar obscuration (Fig. 2) constrain the rotational parameter $j$. For the best fit model of HR 5440 (Fig. 1) we varied the rotation law $V_{\text {rot }}=R^{-j}$ (Fig. 2). For $j>\frac{1}{2}$ the shell effect becomes insufficient to fit the shell absorption for HR 5440. The reason for this is the lower mean velocity shear along the line-of-sight for $j>\frac{1}{2}$. Keplerian rotation is already the best choice to fit HR 5440 .

\section{Interferometric versus Spectroscopic Disk Radii}

Another constraint for the velocity law in Be star circumstellar disks would be the comparison between disk radii derived directly by interferometric methods 


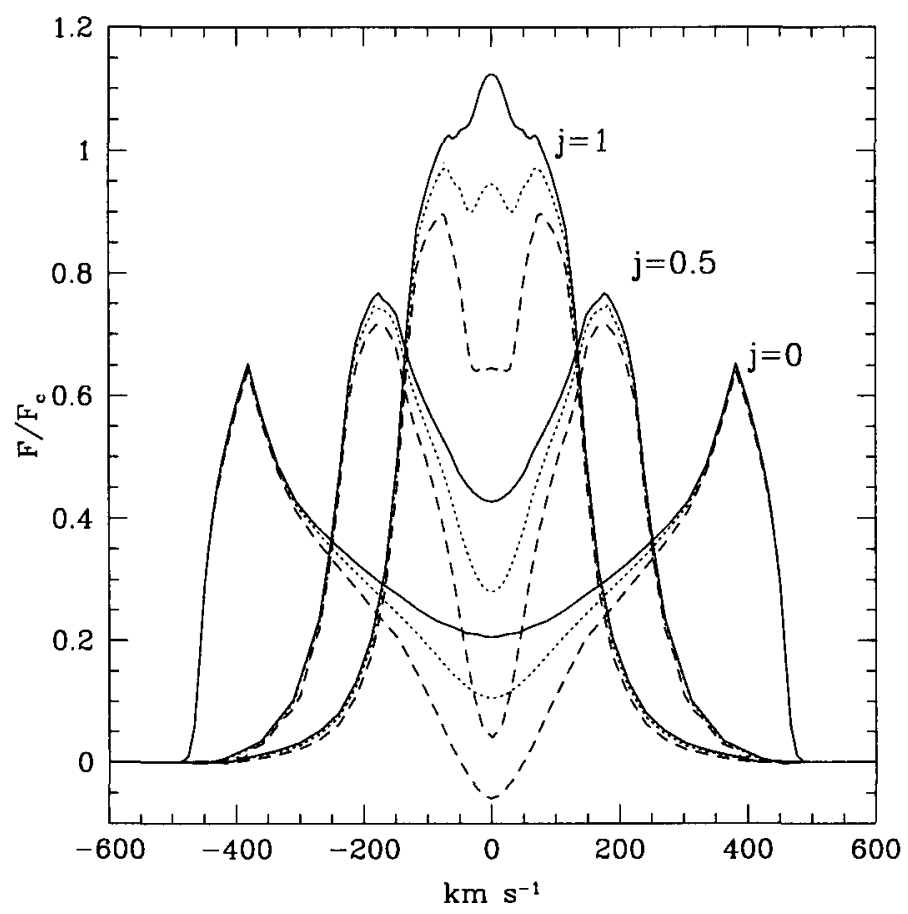

Figure 2. Series of $\mathrm{H} \alpha$ model profiles. The parameters are identical to the best-fit model for HR 5440 (Fig. 1). Profiles for different angular velocity fields $V_{\text {rot }}=423 \mathrm{kms}^{-1} R^{-j}$ are shown. Solid line: without shell absorption and stellar obscuration, dotted line: with stellar obscuration, dashed line: both effects shell absorption and stellar obscuration taken into account.

(Quirrenbach et al. 1997) and disk radii derived from the peak separation of emission lines (Hummel \& Vrancken 1995).

Quirrenbach's disk radii are HWHM, meaning that $a / a_{*}$ has to be considered as a lower limit when compared with emission line peak separations. The uncertainty in the spectroscopic radii is mostly due to the fraction between stellar break-up velocity and observed $v \sin i$, while the velocity at the outer rim of the $\mathrm{H} \alpha$ emission region is well established by the emission peak separation. Therefore spectroscopic radii are also given for the case $v \sin i$ is $80 \%$ of the theoretical break-up velocity (Table 1). Clearly interferometric disk radii are much smaller with respect to spectroscopic radii, which were derived under the assumption of $j=0.5$. We can determine now at which value of $j$ the spectroscopic disk radius matches the interferometric disk radius in $\mathrm{H} \alpha$ :

$$
j=\frac{\log R_{\mathrm{d}} / R_{*}}{2 \log a / a_{*}} .
$$

Since the interferometric radii are lower limits, the estimated values of $j$ (see Table 1) are upper limits. 
Table 1. Columns are the HWHM radii of the $\mathrm{H} \alpha$ visibility by Quirrenbach et al. (1997), spectroscopic $\mathrm{H} \alpha$ emitting disk radii from Hummel \& Vrancken (1995), the same again assuming stellar rotation of $80 \%$ the break-up velocity, the rotational parameter $j$ for both cases of rotation speed of the central star.

\begin{tabular}{cccccc} 
Name & $a^{a)}$ & $R_{\mathrm{d}}$ & $R_{\mathrm{d}}(80 \%)$ & $j$ & $j(80 \%)$ \\
& $a_{*}$ & $R_{*}$ & $R_{*}$ & & \\
\hline$\gamma$ Cas & 6.2 & 11 & 7.0 & 0.66 & 0.54 \\
$\phi$ Per & 6.8 & 22 & 14.1 & 0.81 & 0.69 \\
$\psi$ Per & 9.4 & 46 & 29.4 & 0.85 & 0.76 \\
48 Per & 8.0 & 22 & 14.1 & 0.74 & 0.64
\end{tabular}

\section{Conclusions}

Optically thin emission lines are not useful to derive kinematical constraints. As an example HR 7565 can be well reproduced with $j=\frac{1}{2}$ as well as with $j=1$. For optically thick profiles like HR 5440 the central depression caused by shell absorption and stellar obscuration set more constraints on the model parameters, also on $j$. Model profiles with $j=1$ cannot reproduce shell profiles like that of HR 5440. Only model profiles with $j \simeq \frac{1}{2}$ match the deep shell absorption of HR 5440. We conclude that the formation of $\mathrm{H} \alpha$ shell profiles in Be stars generally requires that $j \simeq \frac{1}{2}$.

Comparing interferometric $\mathrm{H} \alpha$ disk radii with spectroscopic disk radii we find $\bar{j}<0.76$ for $v \sin i=v_{\text {bu }}$ and $\bar{j}<0.65$ for $v \sin i=0.8 v_{\mathrm{bu}}$. We conclude that Keplerian rotation is a valid approximation.

Acknowledgments. Based on observations collected at DSAZ, Calar Alto, Spain and at OHP, CNRS, France

\section{References}

Dachs, J., Hummel, W., Hanuschik, R.W., 1992, A\&AS 93, 116

Horne, K., Marsh, T., 1986, MNRAS 218, 761

Hummel, W., 1994, A\&A 289, 458

Hummel, W., Vrancken, M., 1995, A\&A 302, 571

Quirrenbach, A., Bjorkman, K.S., Bjorkman, J.E., Hummel, C.A., Buscher, D.F., et al., 1997, ApJ 479, 477

Vrancken, M. Butler, K., Becker, S., 1996, A\&A 331, 661 